\title{
Walter Eucken à Paris ?
}

L'introduction de l'ordolibéralisme allemand dans les débats économiques français (1945-1965)

Walter Eucken in Paris? The introduction of German ordoliberalism into French economic debates (1945-1965)

\section{Hugo Canihac}

\section{(2) OpenEdition}

\section{Journals}

Édition électronique

URL : http://journals.openedition.org/ress/3942

DOI : $10.4000 /$ ress.3942

ISBN : $1663-4446$

ISSN : 1663-4446

Éditeur

Librairie Droz

Édition imprimée

Date de publication : 15 décembre 2017

Pagination : 237-263

ISSN : 0048-8046

\section{Référence électronique}

Hugo Canihac, «Walter Eucken à Paris ? », Revue européenne des sciences sociales [En ligne], 55-2 |

2017, mis en ligne le 15 décembre 2020, consulté le 19 janvier 2021. URL : http://

journals.openedition.org/ress/3942 ; DOI : https://doi.org/10.4000/ress.3942 


\title{
WALTER EUCKEN À PARIS? \\ L'INTRODUCTION DE L'ORDOLIBÉRALISME ALLEMAND \\ DANS LES DÉBATS ÉCONOMIQUES FRANÇAIS (1945-1965)
}

HUGO CANIHAC

Sciences Po Bordeaux, Centre Émile Durkheim

hugocanihac@gmail.com

\begin{abstract}
Résumé. Au sein de l'importante littérature qui s'est consacrée ces dernières années à l'exploration des origines intellectuelles des politiques économiques européennes, peu de doctrines ont suscité autant d'intérêt que ce qu'il est convenu d'appeler l'ordolibéralisme allemand. Cet article voudrait inviter à réinterroger la carrière internationale des idées ordolibérales et le récit, souvent quelque peu linéaire, de leur diffusion lente de l'Allemagne à l'Europe. Nous examinerons pour cela l'introduction des idées ordolibérales en France, c'est-à-dire les processus qui leur ont permis de circuler, d'être reçues et discutées dès les années 1950. L'enquête est appuyée sur la collecte de l'archive de cette circulation - traductions, préfaces, comptes rendus, commentaires, etc. -, sur l'identification des configurations d'acteurs impliqués, et sur le repérage des usages qu'ils déploient de ces idées. Nous montrerons ainsi que les idées ordolibérales sont connues en France plus largement que le récit traditionnel ne le laisse penser. Mais, tout en les naturalisant dans le contexte français, cela contribue à les distinguer des usages qu'elles recevaient dans le contexte allemand. La circulation des idées ordolibérales vers la France est donc précoce, mais elle se fait par une hybridation avec les débats économiques français.
\end{abstract}

Mots-clés: histoire de la science économique, histoire sociale des idées, ordolibéralisme, néo-libéralisme.

\begin{abstract}
In the vast literature devoted to the exploration of the intellectual origins of European economic policies, few doctrines have attracted as much interest as the school known as German ordoliberalism. This article investigates the international career of ordoliberal ideas. It aims at questioning the traditional narrative, often somewhat linear, of their slow spread from Germany to Europe. It will examine the introduction of ordoliberal ideas in France- - that is, the processes that led to the diffusion, reception and discussion of these ideas - in the 1950s. The survey is based on the archives of this circulation - translations, prefaces, reviews, comments, etc.- on the identification of the actors involved, and on the identification of the uses they made of these ideas. It will show that ordoliberal ideas were known in France earlier than it is suggested by the traditional narrative. But, whilst acclimatizing them to the French context, this circulation also had the effect of reframing them within French debates.
\end{abstract}

Keywords: history of economics, ordoliberalism, neoliberalism, social history of ideas. 
La crise économique de 2008 et ses conséquences ont renouvelé l'intérêt pour les fondements des politiques économiques menées en Europe. Au sein de l'importante littérature qui s'est consacrée à l'exploration de leurs origines intellectuelles, peu de doctrines économiques ont récemment suscité autant d’intérêt que ce qu'il est convenu d’appeler l'ordolibéralisme allemand (Commun, 2016 ; Schäfer, 2016 ; Fèvre, 2015 ; Solchany, 2015 ; Lechevalier, 2015; parmi les rares analyses plus anciennes en français, Foucault, 2004 [1979]; Bilger, 1964). Ses origines dans l'entre-deux-guerres, les principes libéraux et conservateurs autour desquels il s'organise, son succès politique en Allemagne après 1945 et l'influence exercée par certains de ses partisans dans la construction européenne ont tour à tour fait l'objet d'analyses et de controverses. Malgré les oppositions quant à la nature et aux conséquences exactes des ses idées, l'ordolibéralisme apparaît comme un néo-libéralisme «à l'allemande» (Commun, 20ı6) qui aurait, en toute discrétion, conquis l'Europe jusqu’à en constituer la «tradition cachée»- avant d'être démasqué tardivement (Dardot et Laval, 2009).

Cet article voudrait inviter à réinterroger la carrière internationale des idées ordolibérales et le récit de leur diffusion lente de l'Allemagne à l'Europe. En effet, ces idées n'ont pas été découvertes récemment hors d'Allemagne. Nous le montrerons à partir du cas de la France, elles font en réalité l'objet d'une certaine publicité internationale dans les années 1950. Cela résulte en particulier de l'activité d'économistes qui en discutent la théorie et les implications politiques, au-delà du petit cercle de ceux qui militent aux côtés des ordolibéraux pour un libéralisme nouveau. Pour autant, conclure, à l'inverse, à une «influence » obscure de l'ordolibéralisme sur la pensée économique française depuis les années 1950 n’est guère satisfaisant. S’en tenir à cette hypothèse, ce serait en effet risquer de manquer les usages différents qui sont faits des idées lorsqu'elles circulent dans un nouveau contexte (Skinner, 1969). Ici, l'hypothèse de l'influence ne permet pas de rendre compte des divergences, en particulier au sujet de la construction européenne, qui s'expriment même entre les économistes français les plus proches des idées ordolibérales et leurs collègues allemands (Canihac, 2016; Wegmann, 2002). C'est donc, bien plus, une circulation sans convergence qu'il s'agit de comprendre. 
Nous examinerons pour cela l'introduction des idées ordolibérales, c'est-àdire les processus qui leur ont permis de circuler, d'être reçues et discutées hors d’Allemagne dans les années 1950. La démarche adoptée se veut attentive aux conditions concrètes de la circulation des idées économiques (Matonti, 2012 ; Bourdieu, 2002; voir aussi Hall, 1989). Elle s'attachera à mettre en évidence le contexte politique et scientifique de la circulation, les points de passage et les opérateurs qui la rendent possible, et le travail propre des acteurs qui permettent l'introduction de ces idées - les traductions, recodages et déplacements qui l'accompagnent. L'enquête est appuyée sur la collecte de l'archive de cette circulation - traductions, préfaces, comptes rendus, commentaires, etc. - sur l'identification des configurations d’acteurs impliqués, et sur le repérage des usages qu'ils déploient de ces idées (Vauchez, 20I3).

Après avoir brièvement rappelé les origines et la carrière de l'ordolibéralisme en Allemagne, nous définirons d’abord le contexte dans lequel son introduction s'inscrit en France. Nous mettrons ensuite en évidence le collectif d’acteurs qui permet cette introduction, en soulignant comment ils se positionnent dans ce contexte, avant de dégager pour finir les principaux usages qu'ils font de l'ordolibéralisme à cette période en France. Nous montrerons ainsi que, si les idées ordolibérales sont connues en France plus largement que le récit traditionnel ne le laisse penser, elles subissent des traductions variées lors de leur introduction. Tout en les naturalisant dans le contexte français, cela contribue à les distinguer des usages qu'elles recevaient dans le contexte allemand. La circulation des idées ordolibérales vers la France est donc précoce, mais elle se fait par une hybridation avec les débats économiques français.

\section{LES CONDITIONS D'UNE INTRODUCTION: L'ÉCONOMIE POLITIQUE, SCIENCE DE LA MODERNISATION?}

Si le terme d'ordolibéralisme apparaît en 1950 (Moeller, 1950), les idées qui le caractérisent émergent dès l'entre-deux-guerres en Allemagne. Elles sont formulées par l'économiste Walter Eucken (I89I-1950) et le petit groupe de juristes et d'économistes qui se rassemblent autour de lui à l'université de Freiburg tout au long des années 1930 et 1940. Parmi eux, on compte notamment le 
juriste Franz Böhm (I895-1977), le sociologue Alexander Rüstow (I885-1963) ou l'économiste-moraliste Wilhelm Röpke (I899-1966). Le projet de ce groupe, qui commence à s'exprimer dans une série de publications à partir de 1937 (Böhm et al., 1937), s'inscrit dans le contexte de crise économique et politique de la République de Weimar (Eucken, 1932; Rüstow, 2009). Toutefois, il participe aussi, à la même époque, d'un ensemble de mobilisations en faveur d'un renouveau du libéralisme qui sont, comme la crise du libéralisme, transnationales (Denord, 2007, p. I IO-I I I). Le colloque Lippmann tenu en 1938 à Paris à l’Institut international de coopération intellectuelle en constitue sans doute la meilleure illustration (Audier, 2008). Organisé par le philosophe Louis Rougier autour de la parution du livre La Cité libre du publiciste américain Walter Lippmann, il rassemble des représentants de différents pays qui prônent un libéralisme plus ou moins rénové. Si Eucken n’y est pas présent, on compte notamment parmi les participants Rüstow et Röpke. L’ordolibéralisme s'inscrit donc dans un contexte plus général. Cependant, il possède aussi des traits singuliers.

L’ordolibéralisme est une doctrine complexe. Il se présente comme une théorie économique, dont l’ambition est de refonder le libéralisme économique. Il s'oppose pour cela aux deux grands modèles de théorie (et de politique) économique alors discutés : le marxisme, bien sûr, mais aussi - critique tout aussi essentielle - le libéralisme classique (Eucken, I94I). Pour se différencier du libéralisme «manchestérien», l'ordolibéralisme propose d'encadrer strictement le fonctionnement du marché par des normes juridiques (la «constitution économique»), d’une part, et par des institutions indépendantes (en particulier une banque centrale forte), d'autre part. Cette construction institutionnelle vise à créer un ordre concurrentiel, permettant de prévenir autant les abus de pouvoir de la part des autorités publiques, que ceux de la part des entreprises privées qui seraient tentées de s'établir en monopoles ou en cartels. Ce libéralisme exige ainsi des institutions aux pouvoirs encadrés, mais forts. Il s'articule à une pensée politique et morale conservatrice (Fèvre, 2015 ; Solchany, 2015; Foucault, 2004), et à une conception de la science économique comme science morale (Röpke, 197I). Contre la spécialisation extrême, et l'économie mathématique, la science économique doit, pour les ordolibéraux, 
dialoguer activement avec le droit, la sociologie ou l'histoire, et brouiller les frontières disciplinaires (Audier, 2012; Ptak, 2004).

Si l'ordolibéralisme se présente comme une doctrine scientifique, il revendique dès le départ des applications politiques (Eucken, 2004 [1952], Eucken, 1948). Ainsi, les auteurs qui constitueront le groupe ordolibéral ne se contentent pas de diagnostiquer la crise : ils s'efforcent de la comprendre, et de préconiser des solutions pour y mettre fin. Après 1945, les ordolibéraux - minoritaires dans le paysage des débats économiques allemands - bénéficient de circonstances favorables qui leur permettent de gagner un rôle de premier plan dans la nouvelle République fédérale. Dans un contexte de refondation et de développement rapide de la science économique (Nützenadel, 2005 ; Nörr, 1999), leurs idées sont favorisées par les pouvoirs occupants américains, et à partir de 1948 par le nouveau ministre de l'Économie Ludwig Erhard, qui revendique sa proximité avec les auteurs ordolibéraux (Commun, 2003 ; Mierzejewski, 2006). Il attribue à sa politique - résumée par le mot d’ordre d'économie sociale de marché, forgé par l'économiste et sociologue Alfred Müller-Armack (1947) - le «miracle économique» allemand (Spicka, 2007). Bien que le lien entre le slogan politique et la doctrine ordolibérale ait été vivement contesté (Lechevalier, 2015), Erhard contribue par là à en faire rejaillir les réussites sur ses inspirateurs scientifiques désignés.

C'est au moment de ce triomphe, et alors que le néo-libéralisme se réorganise de façon transnationale après la guerre (avec notamment la création de la Société du Mont-Pèlerin en 1947 - voir Mirowski et Plehwe, 2009), que les idées ordolibérales sont introduites en France. Toutefois, les conditions dans lesquelles s'y déroulent les débats économiques après 1945 sont fort différentes de celles qui prévalent en RFA (Audier, 2012; Fourcade, 2009; Denord, 2007). Pour comprendre cette introduction, il faut alors, en premier lieu, rappeler le contexte politique et académique de ces débats français à cette période. 


\section{I.I. LE TRIOMPHE DE LA PLANIFICATION INDICATIVE}

Comme en Allemagne, les premiers mouvements néo-libéraux avaient vu le jour en France dans le contexte de la crise économique dans l'entre-deux-guerres (Denord, 2007). Le mot lui-même de néo-libéralisme y avait été forgé par l'économiste Bernard Lavergne dans un article de 1932 (Audier, 20I2, p. 4I). L’analyse y était, là aussi, couramment faite de la «crise du capitalisme» et les appels à un renouveau y étaient fréquents. Mais les partisans du libéralisme ne connaissent pas après 1945 un succès politique comparable à celui des ordolibéraux en RFA.

C'est l'idée de « direction » ou de «planification souple » de l'économie qui séduit bien plus largement (Kuisel, 1984). Entendue, de façon générale, comme l'organisation par une instance publique des objectifs et des moyens du développement économique national, elle s'articule au discours sur le nécessaire renouveau des institutions et des élites (Dulong, 1997). Cela justifie la création de nouveaux outils et nouveaux lieux qui doivent donner corps à cette rénovation. Créé en 1946, le Commissariat au Plan est l'un des lieux essentiels de ces efforts (Dulong, 1997; Kuisel, 1984). Il exprime le triomphe d'une vision de l'État actif, doté d'une nouvelle fonction de régulation de la société. Souvent pris pour cible par les proches des ordolibéraux (CEPES, Deutsche Gruppe, 1962), le modèle français se construit sur des bases qui entrent en conflit avec l'économie sociale de marché dont se revendique la RFA.

\section{I.2. LES FRONTIÈRES CONTESTÉES DE LA SCIENCE ÉCONOMIQUE}

Malgré leurs divergences, la politique économique française se veut, comme l'économie sociale de marché en RFA, dotée de fondements scientifiques. À l'image de Pierre Mendès-France, certaines figures politiques plaident ainsi pour une restauration «scientifique» de l'économie française (Rosanvallon, 1987). Mais différentes conceptions de la science économique s'affrontent alors, chez les économistes universitaires, d'une part, et entre les économistes universitaires et un autre groupe d'économistes qui se situe en marge des universités, d’autre part. L'enjeu de ces discussions est de définir ce que doit être la «vraie» science économique, et par là, ce en quoi devra consister une politique économique scientifique. 
Au sein des universités, la science économique française connaît aux environs de 1950 un «tournant générationnel», à l'occasion duquel s'opposent - de façon très schématique - deux groupes principaux (Arena, 2000, p. 969 et suiv.) : les libéraux (par exemple Daniel Villey ou Louis Baudin, mais aussi René Courtin ou Charles Rist), et les «réalistes » qui se proposent de moderniser les conceptions économiques de leurs aînés (André et Jean Marchal, Maurice Byé ou Jean Weiller). Les premiers, dans la continuité de la tradition libérale française, défendent un libéralisme qui est autant philosophique qu'économique. La science économique qu'ils professent se mêle de considérations morales et philosophiques. Les «réalistes», quant à eux, défendent également une conception étendue de la science économique. Elle se veut toutefois moins préoccupée de morale que de sociologie et d'histoire, et se montre attentive aux temporalités et aux institutions sociales qui influent sur le fonctionnement de l'économie. Ce sont donc deux conceptions larges de la science économique, mais qui se déploient dans des directions différentes.

Néanmoins, libéraux comme «réalistes» sont soumis à une concurrence qui, si elle n'était pas totalement nouvelle, prend alors une ampleur inédite. Les revendications d'un nouveau rôle pour l'État dans l'économie s'accompagnent en effet de la montée de la figure de l'ingénieur-économiste, caractérisée par des connaissances plus techniques, plus mathématiques, plus au fait des nouveaux développements théoriques internationaux - et voué à l'économie appliquée au service de l'État (Arena, 2000; Lebaron, 2000, p.93-I03). Or ces compétences sont davantage concentrées dans les lieux para-universitaires, comme Polytechnique, que dans les universités où enseignent, pour la plupart, les libéraux comme les «réalistes» (Fourcade, 2009, p. 205). Cette concurrence entre deux figures de l'économiste - non contradictoires, nous allons le voir - coïncide avec un moment de «crise des vocations » économiques à l'Université : des postes ouverts ne sont pas pourvus faute de candidats en 1950 (Denord, 2007, p. 24I). Face aux conceptions larges de la science économique prônées par les libéraux comme par les «réalistes » à l'Université, les ingénieurs-économistes semblent ainsi revendiquer avec un certain succès pour eux-mêmes la définition de la science économique utile appelée de ses vœux par Mendès-France. 
L'introduction des idées ordolibérales en France, jusqu'à la fin des années 1950, est inséparable de ce double contexte, politique et scientifique. En effet, auréolées des succès de la RFA, elles vont apparaître aux économistes français comme une ressource susceptible de conférer un surcroît de légitimité à leurs conceptions. Tantôt, dans le contexte d'une opposition politique au libéralisme économique, ce sont les usages politiques et les succès revendiqués des thèses ordolibérales outre-Rhin qui seront valorisés. Tantôt, à une période où la science économique française cherche à redéfinir son rôle social et ses frontières, ce sont les options méthodologiques et scientifiques des ordolibéraux qui seront mises en avant. C'est suivant ces deux lignes distinctes que les idées ordolibérales font leur entrée dans les débats hexagonaux.

\section{LES PASSEURS DE L'ORDOLIBÉRALISME EN FRANCE: ENTRE MILITANTS ET RÉFORMATEURS}

L’introduction des idées ordolibérales en France, dans des ouvrages, des traductions, des recensions ou des articles, est le fait d'un petit collectif d'économistes au cours des années 1950. Leur rôle est crucial à plusieurs égards. Tout d'abord, en raison de la division du travail différente qui s'établit entre les disciplines, les juristes sont presque totalement absents - alors même que les ordolibéraux sont, en Allemagne, autant des économistes que des juristes (parmi les rares exceptions, un juriste qui enseigne à l'université franco-allemande de la Sarre: Constantinesco, 1960). Cela explique le sentiment durable d’incompréhensions qui prévaudra entre juristes français et allemands au cours de la construction européenne quant à certaines notions clés de la pensée ordolibérale (à titre d'exemple, voir Institut d'études juridiques européennes, 197I).

De plus, après la guerre, les liens universitaires entre les sciences économiques des deux pays demeurent relativement faibles - malgré quelques exceptions majeures, à l'image de François Perroux, nous allons le voir. L'économiste Robert Mossé constatait en 1963 que «nous sommes encore complètement coupés, au point de vue des relations scientifiques, de l'Allemagne, des économistes allemands et des livres de langue allemande. À part quelques économistes, 
que l'on compte sur les doigts d'une seule main [...] on vit en France dans l'ignorance à peu près totale de la pensée germanique» (Mossé, 1963, p. 526).

Enfin, les ouvrages des «pères fondateurs» ne sont pas traduits en français. Font exception ceux de Röpke, qui bénéficie de traductions dès 1940 de certains de ses ouvrages et enseigne à Genève, où sont publiées la plupart de ses traductions en français. Il faut cependant remarquer à ce titre que deux ouvrages d'Eucken sont, eux, traduits en anglais rapidement après sa mort (Eucken, I95 I ; 1950), sous l'impulsion de Hayek à la London School of Economics (LSE). Laccès aux textes des ordolibéraux demeure cependant complexe pour les lecteurs français. Le rôle des économistes qui les présentent n’en apparaît que plus grand.

Encadré I. Les traductions françaises de W. Röpke jusqu’à la fin des années 1950 Jusqu'à la fin des années 1950 sont traduits:

- Explication économique du monde moderne, 1940 (1937), Paris, Librairie de Médicis (réédité en 1946 et 1951);

- La Crise de notre temps, 1943 (1942), Neuchâtel, Editions de la Baconnière;

- Explication de l'Allemagne, 1945 (1945), Genève, Le Livre Vert;

- «Civitas humana» ou les Questions fondamentales de la réforme économique et sociale: capitalisme, collectivisme, humanisme économique, 1946 (1944), (2e édition), Paris, Librairie de Médicis;

- La Communauté internationale, 1947 (1945), Genève, C. Bourquin;

- L'Économie mondiale aux XIX et $x x^{e}$ siècles, 1959 (196I), Genève-Paris, Droz - Minard, Publications de I'Institut universitaire de hautes études internationales.

Son traducteur attitré en France est Paul Bastier (1874-1955), un spécialiste de littérature allemande qui sera sous-préfet du Bas-Rhin. En Suisse, ce sont des collègues de Röpke à Genève qui prennent en charge la traduction.

Mais ces introducteurs de la pensée ordolibérale ne forment pas un groupe homogène. S’il s'agit pour les uns de défendre une cause politique commune avec les ordolibéraux, l'enjeu est, pour les autres, de réformer la science économique française. 


\section{I. LE PREMIER CERCLE: LES MILITANTS DU NOUVEAU LIBÉRALISME}

Les acteurs de cette introduction sont, en premier lieu, des auteurs liés aux réseaux libéraux constitués dès les années 1930 autour du colloque Lippmann - Jacques Rueff (1896-1978), Louis Baudin (1887-1964)' et Daniel Villey (1910-1968)² notamment. Ils ont tissé des liens directs et parfois étroits avec les ordolibéraux. En raison de son fort investissement par les auteurs liés à l'ordolibéralisme (Bank, 20I3), la Société du Mont-Pèlerin, dont la section française est présidée par Rueff à partir de 1950, est un lieu important où se tissent ces liens. On compte ainsi parmi ses participants français Rueff, Baudin ou Villey - qui tous contribuent à faire connaître les débats d'outre-Rhin en France. Ils sont même «officiellement» reconnus par le groupe ordolibéral, comme l'atteste le fait qu'ils publient dans la revue ORDO: entre 1948 et 1960, c'est le cas de Rueff (en 1959 et 1960), Baudin et Villey.

Ce groupe réunit des économistes aux trajectoires fort différentes. Certains sont des figures de l'Université française. Ainsi de Baudin ou de Villey. Ce dernier est l'héritier d'une famille d'universitaires libéraux - son grand-père, Edmond Villey (I848-1924), fut l'un des fondateurs de la Revue d'économie politique.

I Louis Baudin (1887-1964) étudie le droit à l'université de Paris où il obtient sa thèse en 1910 (La Législation fiscale espagnole), en même temps que le diplôme de l'École libre des sciences politiques. En 1922, il obtient un poste d'économie politique à l'université de Dijon, puis en 1937 à la Sorbonne. II participe au colloque Lippmann de 1938. Baudin est très lié au catholicisme social, dans une version autoritaire illustrée par ses liens au Portugal de Salazar: il préside à partir de 1948 la chambre de commerce franco-portugaise et le conseil d'administration de la banque franco-portugaise à partir de 1958. Auteur d'une œuvre très variée (de la politique du crédit à de nombreuses publications sur l'Empire inca et son économie), il occupe des positions institutionnelles centrales dans l'économie politique d'après-guerre.

2 Daniel Villey (1911-1968) est, comme Eucken, issu d'une famille intellectuelle et universitaire. Après des études de droit et d'économie à Caen, et une thèse publiée en 1936 il est reçu premier à l'agrégation en 1938. Durant les années 1930, il est proche du personnalisme de la revue Esprit, à laquelle il collabore, et des mouvements de «troisième voie» liés au catholicisme social. Durant la guerre, il participe à la Résistance et se rapproche du gaullisme. Nommé à Paris en 1956, il est également membre du Comité technique de l'Institut national d'études démographiques (INED) et vice-président de l'Institut de démographie de l'université de Paris (1957-1958). En 1968, il préside la Société d'économie politique. II est vice-président de la Société du Mont-Pèlerin dans les années 1960 avant d'en prendre la présidence par intérim peu avant sa mort, à la fin de 1967. 
Professeur à la faculté de Paris, il prendra la suite de Baudin à la vice-présidence de la Société du Mont-Pèlerin (Audier, 20ı2, p. I64). Son libéralisme est plein de tensions : s'il est l'un des traducteurs, en 1939, d'un ouvrage dirigé par Hayek et Ludwig von Mises sur L'Économie dirigée en régime collectiviste : étude critique sur les possibilités du socialisme, il avait aussi, en 1936, été un soutien de la politique du Front Populaire (Villey, 1936). Sa pensée s’inscrit dans la filiation philosophique du catholicisme social et des mouvements de «troisième voie » des années 1930 (Audier, 2012, p. 160-162), qui refusent un capitalisme destructeur de la «personne» humaine. Dans les années I930, c'est un collaborateur très régulier de la revue Esprit, et il est étroitement lié aux milieux personnalistes, autour de François Perroux, dont il fréquente le séminaire à l'École pratique des hautes études (EPHE) en 1938-1939 (Cohen, 2012, p. 343). La défense du néo-libéralisme chez Villey est donc inséparable de préoccupations sociales et morales, très marquées par le catholicisme social et «communautaire».

À côté de ce professeur, la carrière de Rueff, singulièrement, représente une tout autre trajectoire : ingénieur, fonctionnaire et enseignant très tôt internationalisé, il se situe aux marges de l'Université, mais au cœur des enjeux pratiques de l'après-guerre. Significativement, dans les «mélanges» publiés en son honneur en 1966, Villey le décrira comme un «prophète» incompris dans son pays, soulignant à la fois la notoriété internationale de l'économiste français, et les limites de sa réception en France - malgré le rôle majeur qu'il jouera dans les réformes économiques mises en œuvre lors du retour du général de Gaulle au pouvoir (Audier, 2012). Le libéralisme prend, chez lui aussi, une dimension fortement morale et philosophique (Rueff, 1966, p. 563). Il n'est pas non plus sans ambiguïtés : farouche opposant de Keynes, il définit sa démarche pour rénover le libéralisme comme la recherche d’un «libéralisme de gauche » ou «libéralisme social» (Audier, 20I2, p. I44-I45). En cela, il est proche d'une autre figure majeure du libéralisme français de l'époque, Maurice Allais, lui aussi ingénieur de formation, participant à la première réunion du Mont-Pèlerin avant de s'en écarter. Comme Rueff, il se réclame d’un libéralisme «social» qui prend la forme d'un «planisme concurrentiel» cherchant, à son tour, un «tiers chemin» entre laisser-faire et socialisme (Diemer, 20I2; Audier, 20I2). 
Malgré leurs trajectoires différentes, ces auteurs se retrouvent autour d'une cause qui est d'abord militante: la défense d'un «nouveau» libéralisme, entamée dès les années d'entre-deux-guerres. Ils sont organisés autour de lieux communs, par l'intermédiaire desquels ils peuvent promouvoir leurs idées. Scientifiquement, ils constituent ainsi un groupe central au sein de la libérale Revue d'économie politique (en 1957 sont notamment membres du Comité de rédaction de la revue Baudin, Villey, Allais, Rueff). À l'articulation des discours académiques et politiques, ils bénéficient également de l'autorité traditionnelle de l’Académie des sciences morales et politiques, et en particulier de sa section d'Économie politique, statistiques et finances (Académie des sciences morales et politiques, 1960) : Baudin en était membre depuis 195 I, tandis que Rueff, qui participe à ses travaux depuis les années 1930, y avait fait son entrée en 1944, et l’avait présidée en 1953.

Une figure atypique qui illustre bien la porosité entre débats savants, milieux «intellectuels » et politiques qui s'établit dans cette arène. Claude-Joseph Gignoux (I890-1968), agrégé d'économie avant 19I4, avait dans les années d'entre-deuxguerres dirigé un journal d’industriels, puis la Confédération générale du patronat français, et été député et sous-secrétaire d'État à l'Économie dans le cabinet Laval (193I-1932). Après avoir largement participé aux instances de direction de l'économie sous le régime de Vichy, il s'était à nouveau tourné après 1945 vers la recherche et l'écriture. Il était le collaborateur régulier depuis 1950, et depuis 1954 le directeur, de la conservatrice Revue des deux mondes. Les liens entre cette revue et l'Académie profitent aux académiciens: Rueff y publie régulièrement au cours des années 1950, de même que Baudin, qui y donne notamment un article sur le «néo-libéralisme» en 1957. Röpke peut aussi, à son tour, publier un article dans la Revue des deux mondes, où il rend compte de la situation allemande en 1959 (Solchany, 20I5, p. 26I). Il est élu membre correspondant de l'Académie des sciences morales et politiques la même année, ce qui lui confère une légitimité certaine en France. Le journal Le Monde rend ainsi compte de l'élection d’un économiste «de grand renom» («L'économiste allemand Wilhelm Röpke est élu correspondant de l'Académie des sciences morales et politiques », 1959). L’introduction de l'ordolibéralisme, et singulièrement de Röpke, s'appuie donc d’abord sur les liens militants avec ses amis français, et les ressources variées dont ils disposent. 


\subsection{LE DEUXIÈME CERCLE:}

\section{LES RÉFORMATEURS DE LA SCIENCE ÉCONOMIQUE}

Mais ces auteurs n'ont pas le monopole de la discussion des ordolibéraux à cette période. Le discours ordolibéral est également introduit par un groupe plus diffus d'économistes «réalistes » qui poursuivent des objectifs différents. Au sein de ce groupe, la connaissance des ordolibéraux est moins liée à un engagement militant qu'à une proximité avec l'Allemagne en général: ainsi de Perroux, qui avait très tôt développé des liens avec la pensée économique d'outre-Rhin (Cohen, 20I2, p. I42), d’André Marchal ${ }^{3}$ et de son frère Jean, qui avaient commencé leur parcours universitaire à Nancy, ou d’André Piettre4, professeur à Strasbourg de 1943 à 1953.

Ils forment eux aussi un groupe hétérogène, qui est cependant animé par une commune ambition réformatrice au sein de la science économique française. Cela se manifeste notamment par la création, autour d’André et Jean Marchal et de Jean Weiller5, de la Revue économique en 1950 (Steiner, 2000, p. IOI2). Elle participe d'un mouvement plus général de renouvellement des sciences sociales, et s'ouvre très largement aux disciplines voisines - notamment à l'école des Annales, par l'intermédiaire de Ferdinand Braudel, qui dirige la $\mathrm{VI}^{\mathrm{e}}$ section de l’EPHE. Elle est d’ailleurs publiée chez Armand Colin, l'éditeur de la jeune Fondation nationale des sciences politiques, avec laquelle leur secrétaire commun, Jean Meynaud, établit le lien (Bermond, 2008, p. I15). C'est

3 André Marchal (1907-1968) soutient une thèse d'économie à Nancy, en 1931, sous la direction de Lucien Brocard. D'abord avocat à la Cour d'appel de Nancy (1929-1935), il enseigne ensuite à l'université de Dijon (1935-1939), puis est chargé de cours à la faculté de Paris (1942-1944) avant d'être agrégé en 1945 et titularisé à Paris. À partir de 1954, il est directeur d'études à l'EPHE. Son frère aîné, Jean Marchal, a un parcours sensiblement analogue. II avait soutenu en 1929 à Nancy sous la direction de Brocard une thèse intitulée Union douanière et organisation européenne.

4 André Piettre (1906-1994) est agrégé (|er) de droit puis professeur à l'université de Strasbourg (1937-1952), avant d'être nommé à Paris en 1953.

5 Jean Weiller (1905-2000) après des études à la faculté de droit de Paris et à l'École libre des sciences politiques, obtient l'agrégation de droit. À partir de 1930, il est conseiller technique pour l'administration économique française (1930-1935), puis enseigne à la faculté de Poitiers (1935-1955) avant d'être nommé à Paris. II est à partir de 1950 directeur d'études à l'EPHE. 
dans le cadre de ce projet scientifique plus général que s'inscrivent les auteurs du «deuxième cercle» de la réception de l'ordolibéralisme en France.

Néanmoins, la création de cette revue souligne bien les relations complexes qui s'établissent entre ce second groupe et les économistes qui appartiennent au premier cercle des ordolibéraux. Elle est placée sous le patronage de deux aînés: le «réaliste» Albert Aftalion (1874-1956) qui la dirige (Arena, 2000, p. 97I), et est lui-même membre du comité de rédaction de la Revue d'économie politique (Steiner, 2000, p. I0I5 et suiv.), et Henri Noyelle, penseur de l'économie dirigée dans les années 1930. La nouvelle revue n’entend pas faire concurrence à la Revue d'économie politique, mais veut «se poser [...] à côté d'elle» (Aftalion, I950, p. I) et s'ouvrir davantage aux sciences sociales comme à l'économie mathématique.

\section{Encadré 2. Devenir un introducteur de la pensée ordolibérale en France: le cas de Raymond Barre (1924-2007)}

Un cas illustre bien la densité de cette nébuleuse - autant en raison de l'importance qu'il prendra par la suite, que du fait de son rôle pionnier dans la présentation des idées ordolibérales aux lecteurs non-germanistes (on va le voir). Raymond Barre ${ }^{6}$, issu d'une famille catholique installée à la Réunion, fait son arrivée en métropole en 1946. II ne participe pas des réseaux déjà constitués avant ou pendant la guerre. Inscrit à la faculté de droit et à l'IEP de Paris, il y fait quatre rencontres qui jouent un rôle décisif dans son orientation (nous nous appuyons ici sur Casanova, 2010, p. 27-31 ; Rimbaud, 2015, p. 36-64). C'est tout d'abord André Piatier, dont il suit les cours à l'IEP et qui lui suggérera son sujet de thèse - thèse dirigée par André Marchal. C'est ensuite

6 Raymond Barre (1924-2007) arrive en métropole en 1946 et suit un cursus de droit et d'économie qui le conduit à l'agrégation en 1950 (il est classé deuxième). D'abord nommé à l'université de Caen, puis de Tunis, il est à partir de 1958 directeur de recherche à la Fondation des sciences politiques, puis professeur à l'IEP de Paris à partir de 1961, et se voit confier une chaire d'économie politique à la faculté de droit de Paris en 1963. II entre en politique comme directeur de cabinet de son collègue Jean-Marcel Jeanneney (UDR), lui-même agrégé d'économie et ministre de l'Industrie et du Commerce du général De Gaulle (1959-1962). En 1966, il fait son entrée au Commissariat général au Plan, puis est nommé vice-président de la Commission européenne en charge des affaires économiques et financières (19671973). Après un passage par la Banque de France, il est nommé ministre du Commerce du gouvernement de Chirac en 1976, puis Premier ministre la même année jusqu'à 1981. 
Perroux, dont il suit le séminaire sur Keynes, à l'IEP également, et qui lui fait découvrir Schumpeter, initiant des liens étroits entre les deux hommes ${ }^{7}$. C'est enfin Raymond Aron, dont il suit un séminaire sur Marx, et avec lequel il noue également des relations durables.

C'est précisément dans ce séminaire que Barre fait la rencontre d'Eric de Dampierre (1928-1998), qui crée en 1952 une collection chez Plon - «Recherches en Sciences Humaines» - sous le patronage de Raymond Aron (Rimbaud, 2015, p.44). La collection a pour but de traduire et de faire connaître des auteurs étrangers, principalement anglophones, aux chercheurs en sciences sociales français. Le deuxième ouvrage de cette collection$^{8}$, Scientisme et sciences sociales de Hayek, est traduit en 1953 par Barre. Son amitié avec Aron conduit aussi le jeune économiste à collaborer avec la revue Critique, fondée en 1946 par Georges Bataille avec le soutien du CNRS (au Comité de la revue siègent en 1953, entre autres, Aron, Braudel, Marjolin). Poursuivant son travail d'introduction d'économistes étrangers, il y publie rapidement sur l'ordolibéralisme (Barre, 1952). Arrivé peu avant à Paris, sa trajectoire à cette période fournit ainsi un exemple des multiples interconnexions qui s'établissent alors entre les différents groupes d'économistes, la théorie et la pratique de l'économie.

On le voit, les introducteurs de l'ordolibéralisme forment un collectif complexe. Les thèses ordolibérales étaient connues par un large éventail d'économistes, du petit nombre des plus militants en faveur du néo-libéralisme, au plus large groupe de ceux qui aspiraient à renouveler la science économique. Or cette introduction en ordre dispersé donne lieu à différents usages des mêmes thèses.

7 Lorsque Barre recevra la Légion d'honneur en 1973, son «parrain» sera Perroux. Les deux autres personnes assistant à la cérémonie sont Eve Barre, sa femme, et la veuve d'André Marchal.

8 Le premier était les Éléments de méthode sociologique de Robert. K. Merton. La collection publiera également Talcott Parsons, Jean Stoetzel ou Léo Strauss. 


\section{LES USAGES DE L'ORDOLIBÉRALISME EN FRANCE: TRADUCTIONS D'UN LIBÉRALISME «À L'ALLEMANDE »}

La diversité des introducteurs de l'ordolibéralisme se reflète dans les usages qui sont faits de cette pensée jusqu'au début des années 1960. Elle fait l'objet d'un double travail de traduction qui reflète les luttes qui traversaient la science économique française. Alors que les uns (le deuxième cercle) mettent en avant leur apport méthodologique, qui appuie leur propre définition de la science économique, les autres (le premier cercle) insistent sur leur libéralisme, et sur les résultats de son application en RFA. La référence ordolibérale est ainsi connue de nombre d'économistes français; mais c'est une ressource qui est diversement mobilisée : loin de révéler une influence uniforme de l'ordolibéralisme sur les économistes français, c'est à donner à voir ces recodages différents que la fin de cet article sera consacrée.

\section{I. L'ORDOLIBÉRALISME COMME MÉTHODE: RENOUVELER LA SCIENCE ÉCONOMIQUE}

Dès le début des années 1950, les auteurs du «deuxième cercle » présentent les ordolibéraux dans la perspective de leur apport méthodologique. Moins que d'un militantisme politique, il s'agit ici de promouvoir la définition de la science économique en faveur de laquelle ces auteurs sont eux-mêmes engagés.

À une période où l'accès aux idées ordolibérales est, pour les Français, très limité, Barre joue un rôle important dans cette présentation. Son intérêt précoce pour l'ordolibéralisme est d’abord orienté par une réflexion méthodologique qui vise à souligner l'apport pratique des travaux des économistes. Le traducteur de l'ouvrage méthodologique de Hayek est l'auteur en 1952 d'un article intitulé «L'analyse économique au service de la science et de la politique économique», dans la revue Critique. Il y rend compte, en même temps que des publications de plusieurs autres économistes, d'un article et des conférences en anglais d'Eucken. C'est la méthode «morphologique» - un aspect fort wébérien de la méthode d'Eucken - qui retient tout son intérêt, ainsi que les conséquences qu'elle implique pour la formulation d’une politique économique scientifique (Barre, 1952, p. 338). Discutant le «décalage qui se mani- 
feste entre la réalité économique et l'économie politique», il plaide pour un «renouvellement de perspective» chez les économistes, et indique que, dans cet objectif, «on ne saurait $[\ldots]$ trouver aujourd'hui de guide plus sûr, ni plus suggestif» qu’Eucken (Barre, 1952, p. 33I-332).

De même, André Marchal discute la méthode d’Eucken dans son ouvrage Méthode scientifique et science économique en 1955. Il souligne lui aussi les mérites de sa conception «morphologique » qui lui paraît un bon moyen d'enrichir et de rendre plus rigoureuse la science économique. Il partage en effet la vision, attribuée à Hayek, d'une économie non «scientiste», qui se préoccupe des éléments extra-économiques afin «de faire servir la science à la politique économique » (Marchal, 1955, p. I7). Dans la même inspiration, Weiller publie à son tour en I953 un article dans la revue Critique où il rend compte de l'ouvrage d'Eucken paru en anglais (la série de conférences qu'il prononçait à la London School of Economics au moment de son décès) et le discute dans la perspective de la situation particulière de la science économique en France (Weiller, 1953). Là aussi, c'est la dimension méthodologique et épistémologique qui est privilégiée.

Ces lectures méthodologiques et avant tout techniques n'excluent pas une appréciation critique du travail des ordolibéraux. Piettre oppose par exemple en 1959 les politiques inspirées d'un vrai économiste - Keynes - et celles inspirées par Erhard, qui n’est en aucun cas un «théoricien» (Piettre, 1959, p. 474). Plus que les prescriptions politiques, c'est donc l'inspiration méthodologique de l'ordolibéralisme qui est retenue et mise au premier plan par ces auteurs. S'ils n'en partagent pas tous les aspects, ils s'attachent à souligner que, chez les ordolibéraux, la science économique se doit de demeurer ouverte aux réalités non économiques. L'insistance sur la méthode leur permet ainsi de mobiliser les auteurs allemands pour légitimer la vision large de la science économique qu'ils défendent contre une conception restrictive et purement mathématique. 


\subsection{L'ORDOLIBÉRALISME COMME PROJET: ENTRE MODÈLE ALLEMAND ET TRADITION FRANÇAISE}

Un usage tout différent de l'ordolibéralisme est déployé par les auteurs du premier cercle. Il s'agit avant tout, pour eux, d'encourager des réformes politiques libérales en France en montrant la contribution de l'ordolibéralisme au «miracle économique» allemand.

Dès 1953, Rueff avait publié dans la Revue des deux mondes un article consacré à la «résurrection de l’Allemagne» (Rueff, 1953), dans lequel il se faisait fort de montrer le fondement scientifique du «miracle économique» allemand. Mais cette présentation est surtout illustrée par la traduction de l'ouvrage-manifeste d’Erhard, La Prospérité pour tous, qui, elle, a lieu dès 1959 (réédité en 1965) soit deux ans seulement après sa parution allemande. Cet ouvrage, co-écrit avec un journaliste de la Frankfurter Allgemeine Zeitung (Ptak, 2004, p. 256), était conçu comme un plaidoyer du ministre de l'Économie en faveur de sa politique. Ce n'était pas son coup d'essai : il avait publié divers textes pour expliquer sa politique depuis 1948. L’un d'entre eux - L’Expansion économique allemande, centré sur l'économie internationale de la RFA - avait été traduit en français en 1953, accompagné d'une préface du futur ambassadeur de RFA en France (Vollrath von Maltzan, I899-1967). Il avait fait l'objet de plusieurs comptes rendus dans Le Monde, dont un long papier nuancé mais critique de Mendès-France (1954). Le succès de La Prospérité pour tous éclipse pourtant de loin celui rencontré par l'ouvrage précédent de Erhard. Le livre est d'abord un succès national en RFA (il connaît huit éditions jusqu'en 1964, sa dernière réédition date de 2009). C'est aussi un succès international: entre 1957 et 1960 il est traduit en sept langues (outre le français, il connaît trois éditions en anglais jusqu'à 1960, une en italien, espagnol, suédois, japonais, finnois).

En France, la publication de l'ouvrage prend un sens politique fort. L'ouvrage paraît chez Plon, éditeur traditionnellement conservateur (Sorel, 2016) qui, sous l'impulsion de son nouvel «homme fort» depuis 1950, Charles Orengo (1913-1974) suit une politique de renouvellement éditorial (Dosse, 20I4; Sorel, 2016, p. 239). C'est dans une nouvelle collection, «Tribune libre », que paraît l'ouvrage d’Erhard. Lancée en 1957, sous la respon- 
sabilité d'Orengo, elle publiait à un rythme très soutenu et avec succès ${ }^{9}$ des ouvrages écrits par «des hommes venus de tous les horizons politiques » afin de «prendre position sur les grandes questions de l'heure (Erhard,1959). C'est une collection d'actualité, d'inspiration souvent polémique. Elle compte parmi ses auteurs, en 1959, Michel Debré, François Mitterrand, Maurice Schumann ou Pierre Mendès-France. De façon générale, le communisme et la guerre d'Algérie sont les deux thèmes principaux qui y sont discutés à cette période : le premier ouvrage qui y paraît, en 1957, est celui d’André Philip, Le Socialisme trahi - attaque contre le gouvernement de Guy Mollet, qui vaudra à Philip d'être exclu de la SFIO en 1958 - tandis qu'y paraît peu après le pamphlet d'Aron sur l’Algérie - La Tragédie algérienne (Sorel, 2016, p. 273). La collection devient ainsi rapidement l'un des lieux où se jouent les controverses majeures de la vie politique française.

C'est donc dans le genre de l'essai polémique grand public, non du traité de science économique, que s'inscrit l'ouvrage d'Erhard en France. Comme le souligne d’ailleurs la Préface donnée par Rueff, il ne s’agit pas «d’un ouvrage de théorie économique ». Ce qui en fait «un ouvrage important» (Rueff, 1959, p.i) dont la lecture est «recommandée» aux Français, ce sont les conséquences politiques des thèses défendues, dont la situation de l'économie allemande est la meilleure preuve (Rueff, I959, p.v). L'éloge de la politique économique allemande est d'autant plus appuyé que, depuis 1958, Rueff défend lui-même une politique libérale auprès du général de Gaulle dont il est le conseiller économique. C’est donc une préface-plaidoyer en faveur du modèle fourni par «l'expérience allemande», qui est présentée à ses lecteurs français. L’alternative, pour la France, est tout simplement «la prospérité pour tous, dans la vérité de l'ordre financier, ou la misère des plus pauvres et l'insécurité de la nation, dans le mensonge de l'inflation» (Rueff, 1959, p.vi).

9 Le texte de présentation de la collection sur ses ouvrages de 1959 revendique trois cent mille exemplaires vendus de l'ensemble de son catalogue en un an et demi d'existence. 
Cette présentation militante prend aussi d'autres formes, plus discrètes. C'est le cas en 1964 avec la publication de l'ouvrage qui servira en France de référence sur le sujet pendant de nombreuses années. Intitulé La Pensée économique libérale dans l'Allemagne contemporaine, l'ouvrage est issu d'une thèse soutenue en I960 par un doctorant de Villey, François Bilger (I934-2010) ${ }^{10}$ et publié dans la collection «Bibliothèque d'économie politique» dirigée par André Marchal. Le caractère unique de l'ouvrage de Bilger à l'époque en fait une source incontournable - un quasi-monopole - pour tout lecteur non germanophone qui voudrait connaître les idées ordolibérales précisément. Il permet à son auteur de s'établir comme référence sur l'ordolibéralisme en France ${ }^{11}$.

La thèse développée par l'auteur-largement en accord avec la vision ordolibérale, selon la méthode de «l'objectivité sympathique » (Bilger, 1964, p. 7) - est appuyée par la présentation de l'ouvrage donnée par Villey. Déplorant l'ignorance des «élites françaises» à l'égard de la pensée allemande, Villey salue d’abord un «grand livre ». Il insiste sur deux éléments. Tout d’abord, la dimension scientifique de l'ordolibéralisme en fait une doctrine généralisable au-delà du contexte allemand. Ainsi, le livre de Bilger a pour vertu de montrer que ce sont en Allemagne «des théoriciens abstraits qui conduisent la marche de la science économique et qui inspirent la politique du gouvernement» (Villey, 1966, p.i-ii). La «révolution libérale» allemande est «une œuvre de l'esprit» avant tout, le fait «d'une poignée de jeunes universitaires, sous la conduite d’un maître éminent» (Villey, 1966, p.iii), que les opportunités de

I0 François Bilger (1934-2010) est issu d'une famille alsacienne catholiqueet conservatrice. Son frère, Pierre Bilger, sera le PDG de l'entreprise Alsthom, et son autre frère, Philippe Bilger, sera avocat général à la Cour d'appel de Paris. Leur père, Joseph Bilger, était un militant autonomiste et clérical alsacien; à la Libération, il est condamné à 10 de travaux forcés et 20 ans d'indignité nationale pour faits de collaboration. Après des études à la Sorbonne, François Bilger obtient l'agrégation de sciences économiques et son doctorat sur la pensée ordolibérale sous la direction de Villey en 1960. II est assistant puis professeur à l'université de Strasbourg de 1959 à 1996, tout en donnant des cours à l'IEP de la même ville. Parallèlement à ses activités universitaires, il est membre de la Commission des comptes et budgets économiques de la Nation (1972-1976), Conseiller de la communauté urbaine de Strasbourg (197|-83) et adjoint au maire de Strasbourg (1977-83). II est aussi membre de la Société du Mont-Pèlerin, de l'Association française de sciences économiques, de l'European Economic Association et du Verein für Socialpolitik.

II C'est ainsi encore l'une des sources majeures de Michel Foucault dans son cours de 1979. 
l'après-guerre ont permis de réaliser sur la «table rase» de 1945. Eucken est décrit, avec sans doute un peu d'envie, comme «une sorte de saint de la pensée économique. Et non moins, un professeur» (Villey, 1966, p. iv).

De façon plus novatrice, Villey soutient que ces idées ne sont pas un corps étranger, mais s'inscrivent dans une tradition française. L'introduction de l'ordolibéralisme n'est donc pas, en ce sens, une importation d’un élément conçu comme extérieur. La pensée d’Eucken est expressément rapprochée de celle de «l'institutionnaliste» (Arena, 2000, p. 97 I et suiv.) Gaëtan Pirou - rapprochement qui permet d'enraciner Eucken dans une tradition plus locale. De même, Erhard est comparé à un «nouveau Turgot» qui a donné un «grand témoignage » des possibilités du libéralisme «au monde (Villey, 1966, p.v-vi). Cette naturalisation à destination d'un public français des thèses allemandes s'exprime aussi par la délicate critique que Villey adresse à Bilger d’avoir un peu surestimé l'originalité propre des ordolibéraux (Villey, I966, p.vii). En définitive, il espère à son tour que «ce livre aidera la France à se délivrer des mirages hexagonaux» (Villey, 1966, p.xvi). Cette présentation fait donc apparaître les idées ordolibérales comme le fruit de la méditation d'économistes théoriciens, qui n'est pas incompatible avec le contexte français : au contraire, elles peuvent être ramenées à des traditions françaises.

\section{CONCLUSION}

Loin d'être inconnus, l'ordolibéralisme et les politiques qui s'en réclament font l'objet d’une publicité notable en France dans les années 1950. Mais cette introduction saccompagne d'une double traduction, qui vise à inscrire les auteurs liés à l'ordolibéralisme dans les enjeux scientifiques et politiques du débat économique français de l'époque. Utilisée pour penser la réforme de la science économique, la pensée ordolibérale apparaît comme d'intérêt principalement méthodologique, et, en définitive, dépolitisée; le travail d'Eucken, en particulier, permet de légitimer une définition étendue de la science économique, qui ne se limite pas à des formules mathématiques. Mobilisée pour promouvoir la réforme de l'économie française, elle est au contraire fortement politisée; ce sont alors les auteurs les plus actifs politiquement qui sont convoqués, principalement Röpke et 
Erhard, et le modèle de l'économie sociale de marché. Ce travail d'introduction ne se résume donc pas à une reconduction à l'identique des idées développées outre-Rhin. Il opère, bien plus, une hybridation entre une pensée économique allemande et le contexte scientifique et politique français de l'époque.

On peut, dès lors, comprendre également certains malentendus et divergences durables entre économistes français et économistes allemands. Il s’agit en effet d'une réception partielle. L’absence des juristes, nous l'avons évoquée, est remarquable, eu égard à l'importance que la réflexion juridique occupe dans la pensée ordolibérale allemande (par exemple Böhm, 1950). L’exigence de stabilité monétaire et l'institution d'une banque centrale indépendante qui l'accompagne - fondamentale pour l'ordolibéralisme - reçoit, elle aussi, peu d'écho en France, malgré l'insistance de Rueff sur ce point (Erhard, 1959, p.iii-iv). Ces particularités de l'introduction en France s'expriment, rapidement et pour longtemps, à travers des divergences marquées au sujet de l'interprétation des traités de Rome (Canihac, 2016 ; Lechevalier, 2015 ; Denord et Schwarz, 20ı0; Wegmann, 2002) : alors que, pour Erhard et Röpke, les Communautés européennes sont l'exemple même d'une organisation non libérale, qui compromet la politique menée en RFA, elles sont présentées en France, par Rueff, Villey ou Allais eux-mêmes, comme des réalisations majeures sur la voie du nouveau libéralisme qu'ils défendent. La redécouverte tardive, en France, de la pensée ordolibérale à l'occasion des débats suscités par la crise économique, permettra alors, peut-être, de mieux saisir les implications d'une pensée qui a profondément marquée la construction européenne. 


\section{BIBLIOGRAPHIE}

ACADÉMIE DES SCIENCES MORALES ET POLITIQUES, 195I, Notices biographiques et bibliographiques des membres titulaires et libres et associés étrangers, Paris, Sirey.

-, 1960, Notices biographiques et bibliographiques des membres titulaires et libres et associés étrangers, Paris, Sirey.

AFTALION A., 1950, «Présentation», Revue économique, I-I, p. I-2.

ARENA R., 2000, «Les économistes français en 1950», Revue économique, 5I-5, p. 969-1007.

AUDIER S., 2008, Le Colloque Lippmann: aux origines du néolibéralisme, Lormont, Le Bord de l'Eau.

-, 2012, Néo-libéralisme(s): une archéologie intellectuelle, Paris, Grasset.

BANK M., 2013, Stunde der Neoliberalen? Politikberatung und Wirtschaftspolitik in der Ära Adenauer, thèse soutenue à l'université de Cologne [en ligne]: $<$ http://kups.ub.uni-koeln.de/584l>.

BARRE R., 1952, «L'analyse économique au service de la science et de la politique économique», Critique, 59-7, p. 332-346.

BERMOND D., 2008, Armand Colin: histoire d'un éditeur de 1870 à nos jours, Paris, Armand Colin.

BILGER F., 1964, La Pensée économique libérale dans l'Allemagne contemporaine, Paris, LGDJ.

BÖHM F., 1950, Wirtschaftsordnung und Staatsverfassung, Verlag J. C. B. Mohr, Tübingen.

-, EUCKEN W. et GROSSMANN-DOERTH, H., 1937, «Unsere Aufgabe», Ordnung der Wirtschaft, I, p.VII-XXI.

BOURDIEU P., 2002, «Les conditions sociales de la circulation internationale des idées», Actes de la Recherche en Sciences Sociales, 145, p.3-8.

CANIHAC H., 2016, «(Néo-)libéralisme contre (néo-)libéralisme? Controverses économiques sur les débuts de la construction européenne en France et en Allemagne (1950-1960)», Trajectoires, 10, [en ligne]:

$<$ http://trajectoires.revues.org/2087>. 
CASANOVA J.-C., 2010, «De l'économie politique à la politique économique», in M. Albert, J-Cl. Casanova (dir.), Raymond Barre: un homme singulier dans la politique française, Paris, Éditions de Fallois, [s.p.].

CEPES, DEUTSCHE GRUPPE, 1962, «Frankreichs Wirtschaftsplanung und ihre Bedetung für die EWG», Europa-Archiv, 14.

COHEN A., 2012, De Vichy à la Communauté européenne, Paris, PUF.

COMMUN P., 2003, «La conversion de Ludwig Erhard à l'ordolibéralisme (19301950)», in Patricia Commun (dir.), L'Ordolibéralisme allemand, Cergy-Pontoise, CIRAC/CICC, p. 175-219.

-, 2016, Les Ordolibéraux: histoire d'un libéralisme à l'allemande, Paris, Les Belles Lettres.

CONSTANTINESCO L.-J., 1960, «La constitution économique de la République Fédérale allemande», Revue économique, II-2, p. 266-290.

DARDOT P. et LAVAL, C., 2009, La Nouvelle Raison du monde, Paris, La Découverte.

DENORD F., 2007, Néo-libéralisme version française : histoire d'une idéologie politique, Paris, Demopolis.

- et SCHWARZ, A., 2010, «L'économie (très) politique du traité de Rome», Politix, 89, p. 35-56.

DIEMER A., 2012, «Aspects théorique et pratique de la concurrence dans l'œuvre de Maurice Allais», Cahiers d'économie Politique / Papers in Political Economy, 62-I, p.75-II5.

DOSSE F., 2014, Les Hommes de l'ombre: portraits d'éditeurs, Paris, Perrin.

DULONG D., 1997, Moderniser la politique: Aux origines de la Ve République, Paris, L'Harmattan.

ERHARD L., 1959 (1957), La Prospérité pour tous, Paris, Plon.

EUCKEN W., 1932, «Staatliche Strukturwandlungen und die Krisis des Kapitalismus», Weltwirtschaftliches Archiv, 36, p. 297-321.

-, 1941, Die Grundlagen der Nationalökonomie, Jena, Gustav Fischer Verlag.

-, 1948, «Das Ordnungspolitische Problem», ORDO: Jahrbuch für die Ordnung von Wirtschaft und Gesellschaft, I, p.56-90. 
-, 1950, The Foundations of Economics. History and Theory in the Analysis of Economic Reality, London, William Hodge \& Co.

-, 1951, The Unsuccessful Age or, the Pains of Economic Progress, Edinburgh, William Hodge \& Co.

-, 2004 (1952), Grundsätze der Wirtschaftspolitik, Mohr Siebeck.

FÈVRE R., 2015, «Retour sur le libéralisme conservateur de Wilhelm Röpke», Revue européenne des sciences sociales, 53-2, p. 147-190.

FOUCAULT M., 2004, Naissance de la biopolitique (Cours au Collège de France, 1978-1979) Paris, Gallimard.

-, 2009, Economists and societies: Discipline and profession in the United States, Britain, and France, 1890s to 1990s, Princeton, Princeton University Press.

HALL P.A. (ed.), 1989, The Political Power of economic ideas, Princeton, Princeton University Press.

INSTITUT D'ÉTUDES JURIDIQUES EUROPÉENNES, I97I, La Constitution économique européenne, Actes du cinquième colloque sur la fusion des Communautés Européennes, Liège, IEJE.

KUISEL R., 1984, Le Capitalisme et l'État en France - Modernisation et dirigisme au $x x^{e}$ siècle, Paris, Gallimard.

Le Monde, 1959, «L'économiste allemand Wilhelm Röpke est élu correspondant de l'Académie des sciences morales et politiques », 15 avril.

LEBARON F., 2000, La Croyance économique: les économistes entre science et politique, Paris, Éditions du Seuil.

LECHEVALIER A., 2015, «Eucken under the Pillow: The Ordoliberal Imprint on Social Europe», in Arnaud Lechevalier et Jan Wielgohs (éds), Social Europe: a Dead End?, Copenhague, DjOf Publishing, p. 49-103.

MARCHAL A., 1955, Méthode scientifique et science économique, Paris, Librairie de Médicis.

MATONTI F., 2012, «Plaidoyer pour une histoire sociale des idées politiques», Revue d'histoire moderne et contemporaine, 59-5, p. 85-104. 
MENDÉS-FRANCE P., 1954, «Le redressement économique allemand: politique libérale ou politique réaliste?», Le Monde, 8 juin.

MIERZEJEWSKI A., 2006, «Water in the Desert? The Influence of Wilhelm Röpke on Ludwig Erhard and the Social Market Economy», Review of Austrian Economics, 19, p. 275-287.

MIROWSKI P. et PLEHWE D. (éds), 2009, The Road from Mont-Pèlerin: The making of the neoliberal thought collective, Cambridge, MA, Harvard University Press.

MOELLER H., 1950, «Einzelbesprechungen, Ordo, Jahrbuch für die Ordnung von Wirtschaft und Gesellschaft, Band I, Bd. 2», Jahrbücher für Nationalökonomie und Statistik, 162-2, p. 128-133.

MOSSE R., 1963, Revue d'Économie politique, 73-3, p. 525-527.

MÜLLER-ARMACK A., 1947, Wirtschaftslenkung und Marktwirtschaft, Hamburg, Verl. f. Wirtschaft u. Sozialpolitik.

NÖRR K.W., 1999, Die Republik der Wirtschaft Recht, Wirtschaft und Staat in der Geschichte Westdeutschlands, Tübingen, Mohr Siebeck.

NÜTZENADEL A., 2005, Stunde der Ökonomen. Wissenschaft, Politik und Expertenkultur in der Bundesrepublik 1949-1974, Göttingen, Vandenhoeck \& Ruprecht.

PTAK R., 2004, Vom Ordoliberalismus zur Sozialen Marktwirtschaft - Stationen des Neoliberalismus in Deutschland, Wiesbaden, Springer Fachmedien.

RIMBAUD C., 2015, Raymond Barre, Paris, Perrin.

ROSANVALLON P., 1987, «Histoire des idées keynésiennes en France», Revue française d'économie, 2-4, p. 22-56.

RUEFF J., 1953, «La résurrection de l'Allemagne», Revue des deux mondes, Juin (deuxième quinzaine) , p. 594-608.

-, 1959, «Préface», in ERHARD, 1959 (1957), p. I-VI.

-, 197I, A Humane Economy - the Social Framework of the Free Market, Indianapolis, Liberty Fund ed.

RÜSTOW A., 2009, Die Religion der Marktwirtschaft, Freiburg, LIT Verlag. ed, Walter Eucken Archiv. 
SCHÄFER D., 2016, «A Banking Union of Ideas? The Impact of Ordoliberalism and the Vicious Circle on the EU Banking Union», Journal of Common Market Studies, 54-4, p. 961-980.

SKINNER Q., 1969, «Meaning and Understanding in the History of Ideas», History and Theory, 8-1, p. 3-53.

SOLCHANY J., 2015, Wilhelm Röpke, l'autre Hayek: aux origines du néolibéralisme, Paris, Publications de la Sorbonne.

SOREL P., 2016, Plon: le sens de l'histoire (1833-1962), Rennes, Presses Universitaires de Rennes.

STEINER Ph., 2000, «La Revue économique 1950-1980. La marche vers l'orthodoxie académique?», Revue économique, 5I-5, p. 1009-1058.

VAUCHEZ A., 2013, «Le prisme circulatoire. Retour sur un leitmotiv académique», Critique internationale, 2-59, p. 9-16.

VILLEY D., 1936, «Pour éviter les casse-cou», Esprit, 4-46, p 602-606.

-, 1966, Notes de philosophie économique, Cours de 1965-1966 à la faculté de droit et de sciences économiques de Paris, Paris, Les Cours de Droit.

WEGMANN M., 2002, Früher Neoliberalismus Und Europäische Integration. Baden-Baden, Nomos Verlag.

WEILLER J., 1953, «L'économiste au travail, à l'âge des insuccès», Critique, 9-73, p. 554-562. 